Meta

Journal des traducteurs

Translators' Journal

\title{
Organisation des outils de base des services linguistiques d'une organisation internationale
}

\section{Françoise Cestac}

Volume 25, numéro 1, mars 1980

La documentation

URI : https://id.erudit.org/iderudit/002210ar

DOI : https://doi.org/10.7202/002210ar

Aller au sommaire du numéro

Éditeur(s)

Les Presses de l'Université de Montréal

ISSN

0026-0452 (imprimé)

1492-1421 (numérique)

Découvrir la revue

Citer cet article

Cestac, F. (1980). Organisation des outils de base des services linguistiques d'une organisation internationale. Meta, 25(1), 58-67.

https://doi.org/10.7202/002210ar d'utilisation que vous pouvez consulter en ligne. 


\title{
Organisation des outils de base des services linguistiques d'une organisation internationale
}

\author{
Françoise Cestac \\ directeur, \\ Division de traduction, O.N.U.
}

«Mal utiliser les mots témoigne d'un mépris pour l'humanité. »

Cette citation de Dag Hammarskjöld définit admirablement l'un des premiers soucis de la Communauté internationale : encourager la compréhension et les relations pacifiques entre les peuples. Les mots, leur caractère antique, surtout s'ils sont utilisés d'une culture à l'autre, constituent l'élément essentiel de la communication au sein d'une instance internationale telle que l'Organisation des Nations unies.

Les conférences et réunions de diverses matières constituent une des activités essentielles d'une Organisation réunissant 152 pays de cultures et de langues différentes qui communiquent néanmoins par l'intermédiaire de six langues officielles : l'anglais, l'arabe, le chinois, l'espagnol, le français et le russe.

Deux instruments de travail sont à la disposition de ces nations : l'interprétation et la traduction. La parole et l'écrit sont étroitement mêlés. La parole n'est pas éphémère, elle est durable car enregistrée. L'écrit, par son essence même, est consigné sur un support, le papier, à qui nous donnerons ses lettres de noblesse, en l'appelant documentation.

Afin de mieux répondre à la question posée : « organisation des outils de base des services linguistiques d'une organisation internationale », je préférerai «situer » la documentation dans notre « milieu linguistique », c'est-à-dire au sein de la Division de traduction et en amont des services de traduction qu'il doit servir, dans ce qu'il convient d'appeler le «service de documentation et de terminologie ». suivants.

Avant de décrire ses activités, il convient de rappeler les organigrammes

Dans l'organigramme du Département des Conférences, nous soulignerons le rôle essentiel du «Service de contrôle des documents» que je comparerais à une "gare de triage ». Ce service est chargé d'organiser l'élaboration d'un document, à tous les stades, depuis sa rédaction jusqu'à sa distribution, et de fixer les délais à l'intention de tous les services intéressés, depuis notre Service de documentation et de terminologie jusqu'à celui de la distribution. 


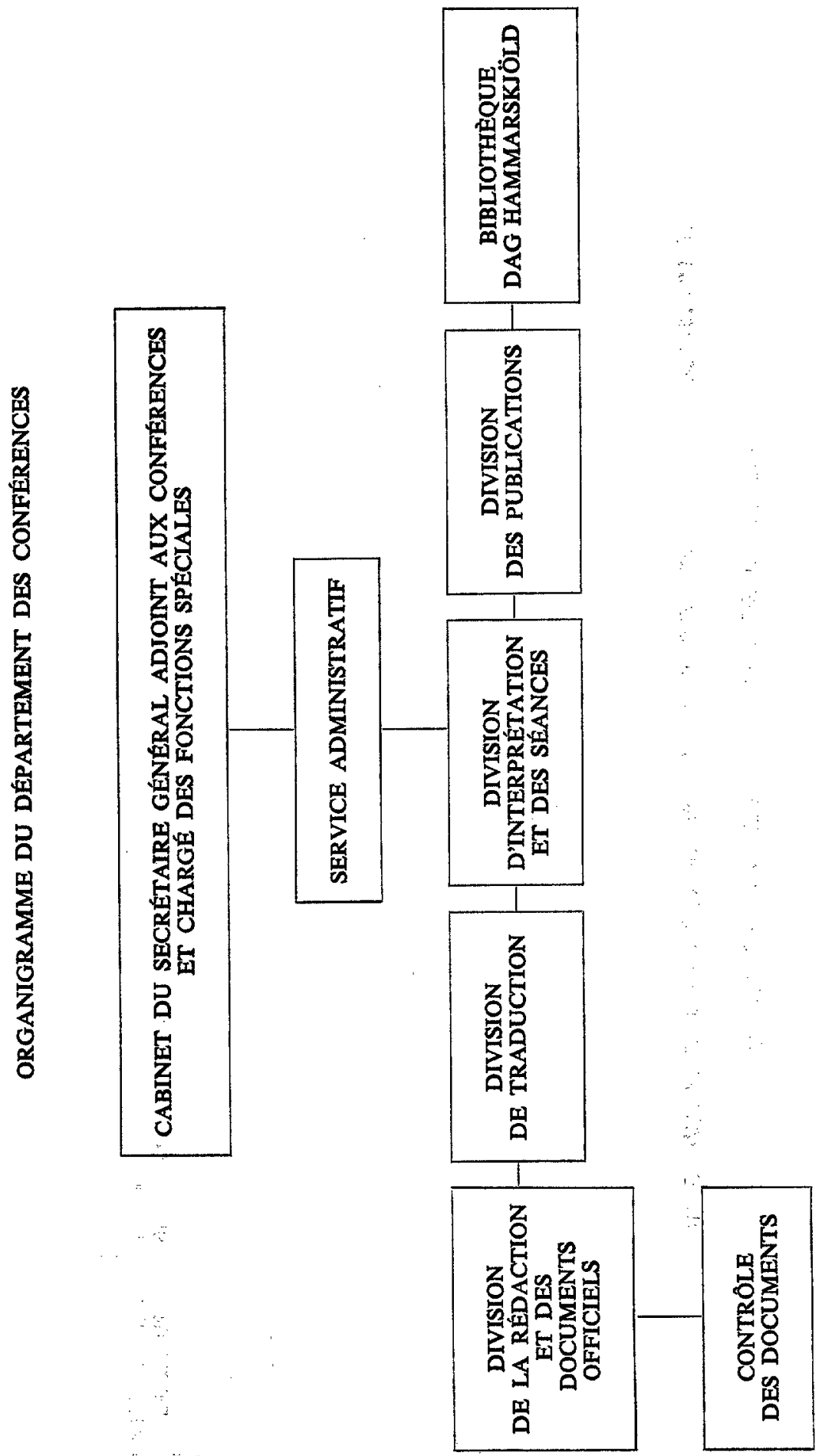




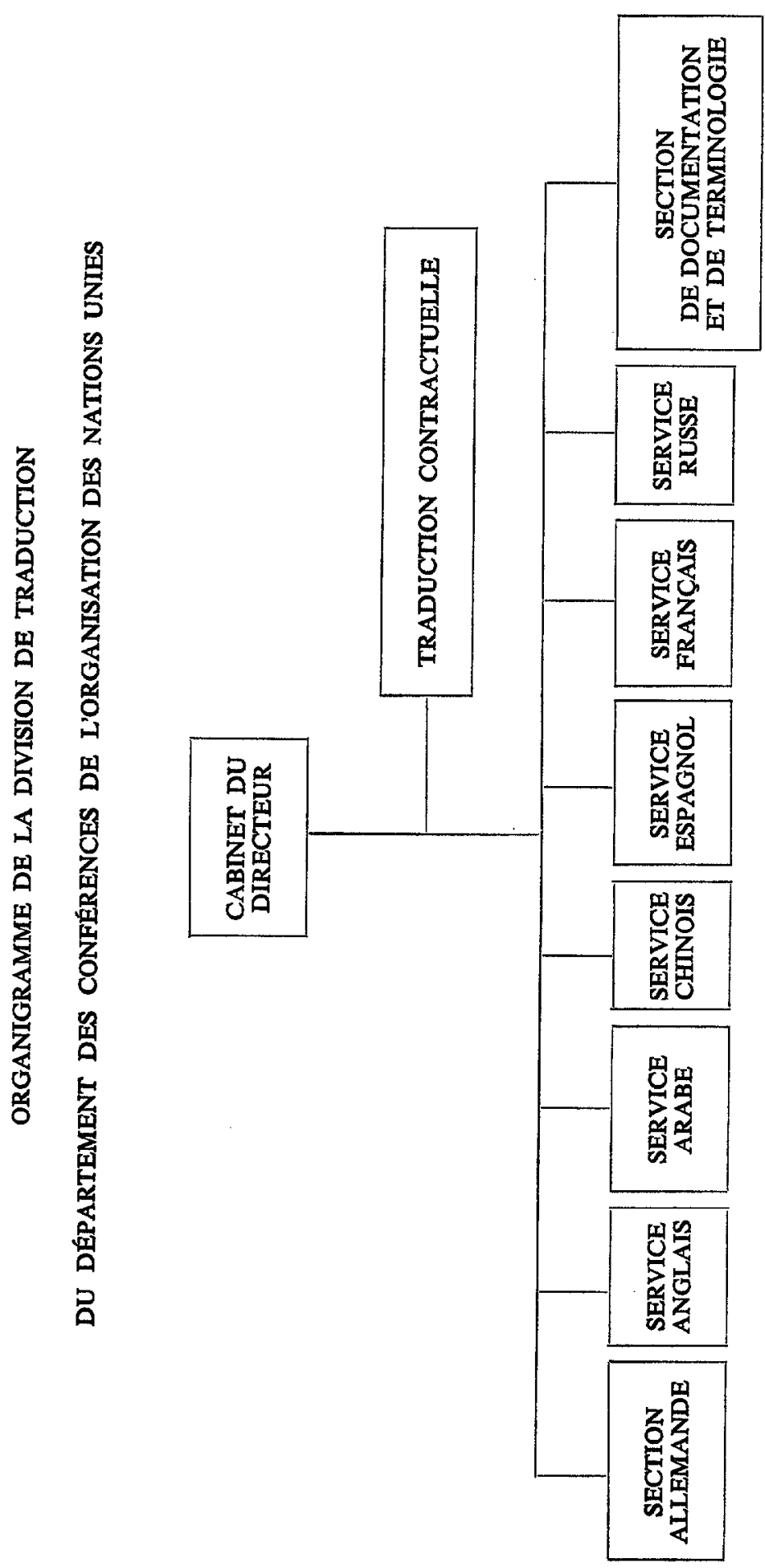




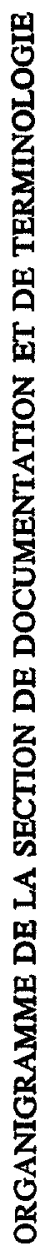

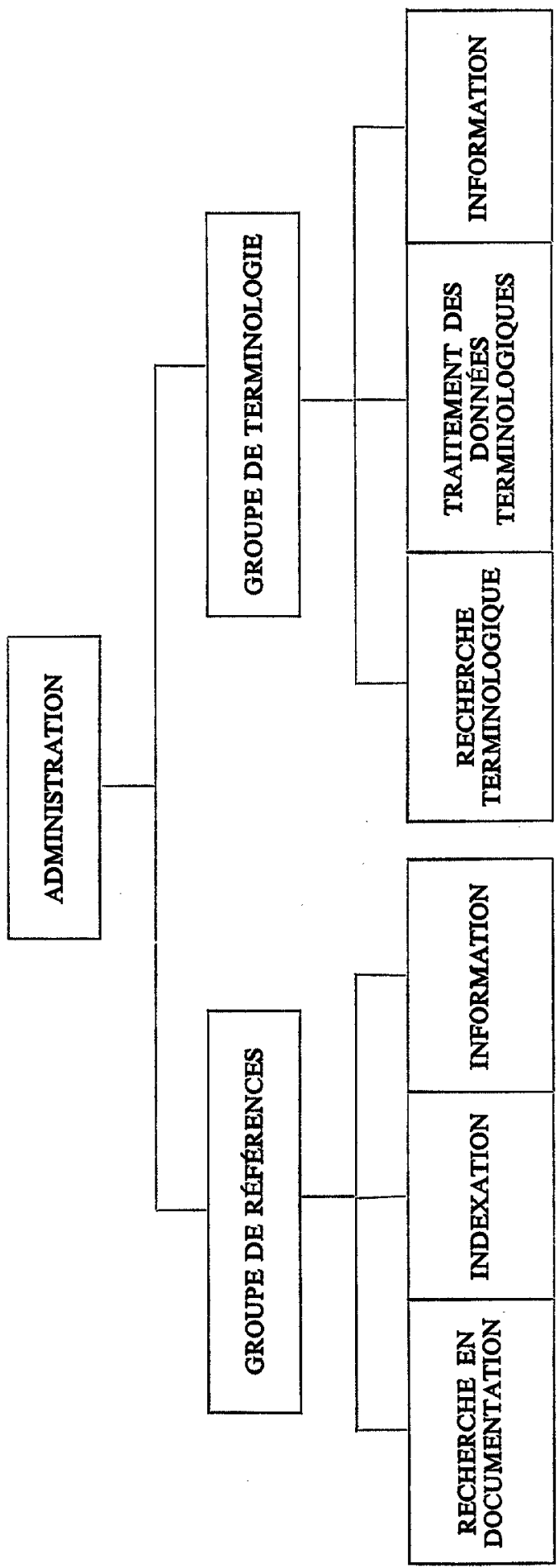




\section{Le Service de documentation et de terminologie}

Les services de traduction de l'Organisation des Nations unies sont particulièrement tributaires des travaux de référence des documents et de la recherche terminologique. Ces services d'appui, qui font partie intégrante des services de traduction dans toute organisation internationale ou intergouvernementale, revêtent une importance capitale à l'Organisation des Nations unies, où les précédents établis et suivis publiés dans des textes qui contiennent des termes précis et concordants reçoivent l'attention du monde entier.

Les travaux de référence des documents et la recherche terminologique effectués par les membres du Service de documentation et de terminologie contribuent à favoriser, faciliter et contrôler la continuité et la concordance de la terminologie utilisée, ce qui est essentiel dans les publications multilingues des Nations unies.

\section{Groupe de la documentation}

Après avoir été rédigés, mais avant d'être traduits, tous les documents de l'ONU sont soigneusement et rigoureusement examinés par le Groupe de la documentation souvent appelé Groupe des références.

Le travail de ce Groupe consiste essentiellement en la préparation, en un temps record, des documents destinés à la traduction. Son objectif principal est de fournir aux traducteurs et aux réviseurs les informations documentaires nécessaires en vue d'assurer l'uniformité et la précision de la traduction. En outre, il permet à ceux-ci d'avoir l'assurance que leur travail ne fera pas double emploi avec la documentation déjà publiée par l'Organisation des Nations unies sur la question et n'ira pas non plus à l'encontre de cette documentation.

La recherche documentaire au niveau du Groupe des références suppose donc :

- d'une part, une recherche bibliographique poussée dans le but de vérifier les titres, tableaux, têtes de chapitres, points de l'ordre du jour, déclarations, études, projets, réunions, séminaires, etc., à partir des rapports, programmes et budgets préparés par les Nations unies et les Institutions spécialisées. Cette recherche est grandement facilitée par la constitution d'une collection de documents publiés par les Nations unies et les Institutions spécialisées (quotidiennement mise à jour) ;

- d'autre part, l'indexation de tous les documents, publiés principalement par les Nations unies, sur fiches manuelles, afin d'extraire au plus vite l'information documentaire ;

- la fourniture aux traducteurs d'exemplaires de textes de références pertinents ainsi que de traductions déjà publiées.

Alors que le Service chinois et le Groupe allemand assurent leurs propres références, le Groupe des références dessert les services anglais, arabe, espagnol, français et russe. Il assure la référence en ce qui concerne pratiquement tous les documents de l'Organisation des Nations unies destinés à être traduits, notamment les traités et accords internationaux dont le Secrétaire général est le dépositaire. 
Pour être en mesure d'assurer ces services, le Groupe des références dispose d'une vaste collection de documents. Il conserve les publications courantes de l'Organisation des Nations unies pendant environ six ans; les documents officiels publiés depuis la création de l'Organisation sont conservés dans ses dossiers. Des exemplaires de tous les textes des traités bilatéraux et multilatéraux qui sont entrés en vigueur et qui sont enregistrés auprès de l'Organisation des Nations unies, les index publiés par la Bibliothèque Dag Hammarskjöld, et les principaux documents des institutions spécialisées ainsi que d'autres organes du système des Nations unies sont également disponibles. Des fichiers permettent de retrouver rapidement et facilement ces documents ainsi que les informations enregistrées sur microfiches.

Le premier groupe est donc chargé de procéder à une « recherche d'antériorité » sur des documents existants afin de faire essentiellement gagner du temps au traducteur.

\section{Groupe de terminologie}

Le second groupe ou Groupe de terminologie a une mission différente, qui se situe dans le domaine linguistique pur. Néanmoins, les terminologues sont aussi des utilisateurs et des producteurs de documentation.

Une fois que le traducteur reçoit le document référencé, son travail est encore facilité par les données et les indications terminologiques sûres dont il dispose. $\mathrm{La}$ terminologie, discipline qui trouve ses origines dans la linguistique, la logique et les sciences de l'information, et qui à la fois respecte et normalise les usages, est essentielle pour assurer la concordance des termes fondamentaux utilisés dans les langues officielles de l'Organisation des Nations unies.

Les terminologues de l'Organisation des Nations unies, qui travaillent dans leur langue maternelle et dans des domaines choisis en fonction des besoins qui, selon les prévisions, seront ceux de l'Organisation, analysent systématiquement les travaux de recherche, les publications et autres documents spécialisés en vue de se familiariser avec les modifications, les variations et les nouveautés dans le domaine $\mathrm{du}$ vocabulaire scientifique et technique.

Afin de donner un exemple du rôle essentiel que joue la documentation en terminologie, je souhaiterais expliquer comment s'effectue la recherche terminologique et sur quelles sources de documentation elle se fonde.

Le travail terminologique est essentiellement orienté vers trois types de recherche :

- la recherche thématique, principalement axée sur la préparation des bulletins de terminologie publiés périodiquement par la Section;

- la recherche ponctuelle, qui vise à résoudre des problèmes spécifiques de terminologie ;

- la recherche systématique, dont le but est de suivre l'évolution de l'activité créatrice du langage et de repérer ainsi les structures lexicales et syntaxiques nouvelles. 
Ces travaux de recherche dépendent toutefois de la qualité de la recherche documentaire sur laquelle ils s'appuient, tant il est vrai que la documentation constitue la matière première par excellence de la recherche terminologique. Les rapports de la terminologie à la documentation pourraient en fait être esquissés en quatre grandes étapes :

\section{CONSTITUTION DE LA DOCUMENTATION}

La recherche documentaire sert de base à l'établissement du corpus. Cet ensemble limité d'éléments sur lesquels se fonde toute étude terminologique est constitué par un corpus d'analyse (dépouillé aux fins des besoins terminologiques) et un corpus de référence (utilisé afin d'y puiser ou vérifier des renseignements d'ordre linguistique ou encyclopédique). Il permet d'établir une nomenclature aussi vaste que possible, basée sur des sources écrites aussi bien qu'orales.

\subsection{Sources écrites}

Les sources consultées par le Groupe de terminologie lors de la préparation d'un bulletin de terminologie sont de deux sortes :

1.1.1 Sources secondaires constituées par des bibliographies, catalogues, inventaires, tels que :

- système d'information bibliographique des Nations unies (UNBIS) ;

— système d'information documentaire des Nations unies (UNDIS);

- Bibliographie internationale des dictionnaires techniques monolingues et multilingues (publiée par l'UNESCO);

- The Subject Guide to Books in Print (publié par R.R. Bowker Company aux États-Unis) ;

- la Bibliographie de la France;

- l'Index des documents des Nations unies (UNDOC) ;

- le catalogue de la Bibliothèque Dag Hammarskjöld aux Nations unies et des bibliothèques et centres de documentation des départements organiques de l'Organisation ;

- les inventaires des travaux de terminologie (ceux de la Banque de terminologie du Québec et de l'AFTERM en particulier);

- les catalogues des éditeurs et les annuaires professionnels, etc.

1.1.2 Sources primaires générales - Cette documentation consiste essentiellement en une collection d'ouvrages de base destinés à résoudre soit des problèmes qui touchent à la langue générale, qu'il s'agisse de l'anglais, de l'arabe, du chinois, de l'espagnol, du français ou du russe (les six langues officielles de l'Organisation des Nations unies), soit des questions d'ordre encyclopédique. Cette collection se compose principalement de :

- dictionnaires généraux (de langue, encyclopédiques ou mixtes);

- dictionnaires et lexiques unilingues spécialisés ;

- dictionnaires et lexiques spécialisés (bilingues ou plurilingues) ;

- encyclopédies ; 
- fichiers de terminologie (ceux des Nations unies et de la Banque de terminologie du Québec entre autres);

- normes (ISO, AFNOR en particulier);

- publications officielles (lois et règlements, arrêtés ministériels en matière de terminologie, mises en garde de l'Académie française, etc.);

- thésauri et classifications, etc.

1.1.3 Sources primaires spécialisées - Il s'agit essentiellement de documents et de publications ayant un rapport direct avec les multiples activités de l'Organisation des Nations unies que ce soit dans le domaine politique, économique ou social. Une telle documentation comprend principalement :

- la charte des Nations unies;

- les résolutions et décisions adoptées par les principaux organes de l'Organisation des Nations unies, ainsi que tout document mentionné dans ces résolutions et décisions ;

- les comptes rendus et rapports officiels ;

- les publications de l'Organisation;

- les documents officiels et les publications des institutions spécialisées du système des Nations unies (UNESCO, OMS, FAO, etc.) ;

- les manuels, journaux, périodiques et monographies spécialisés.

\subsection{Sources orales}

Les sources orales peuvent aussi constituer un outil de base précieux en matière de recherche documentaire et par là même de recherche terminologique. Les contacts étroits entre terminologues, traducteurs, éditeurs et surtout spécialistes des disciplines dans lesquelles s'effectue la recherche terminologique (à savoir, les fonctionnaires des services organiques de l'Organisation des Nations unies, les ingénieurs, techniciens, professeurs, chercheurs travaillant pour l'Organisation ou à l'extérieur) permettent en effet d'établir un réseau d'informateurs qualifiés dont dépendent la qualité et la rigueur du travail terminologique. C'est ainsi que lors de la préparation du *Bulletin de terminologie consacré à la Science et à la Technique au service du Développement », l'établissement du corpus d'analyse et de référence (cf. Tableau - La recherche terminologique au sein de l'ONU) a été grandement facilité grâce aux précieux conseils des spécialistes de la Conférence des Nations unies sur la science et la technique au service du développement.

\section{EVAluation DE LA DOCUMENTATION}

Trois critères d'évaluation ont été retenus par la Section de documentation et de terminologie :

(a) les indications méthodologiques (établissement de la bibliographie, par exemple) ;

(b) la structure de l'ouvrage (systématisation des notions, structuration des renvois, index des mots, etc.);

(c) l'intention de l'ouvrage : vocation informative. 
LA RECHERCHE TERMINOLOGIQUE AU SEIN DE L'ONU

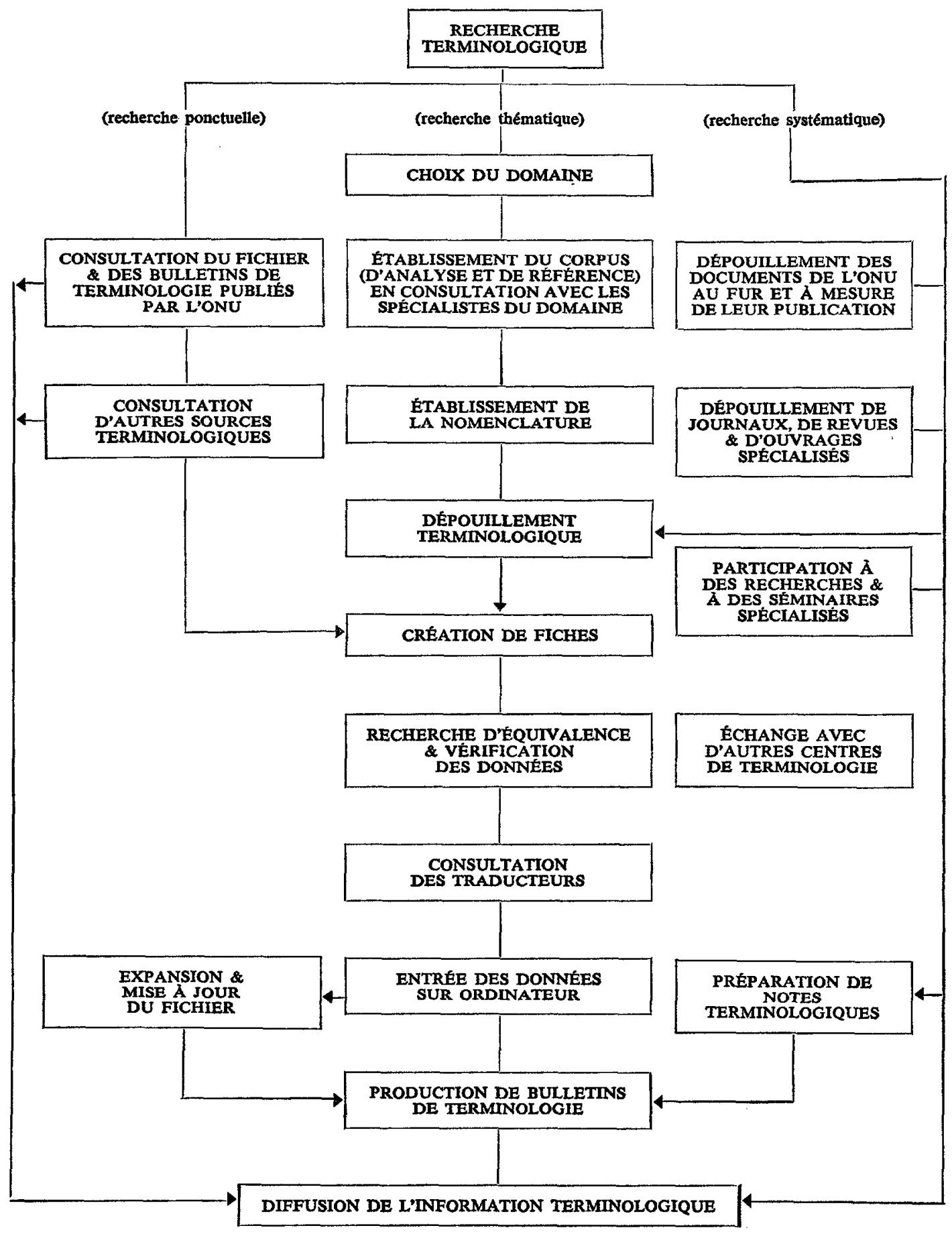




\section{CLASSEMENT DE LA DOCUMENTATION}

Le classement de la documentation terminologique s'effectue par pôles de regroupement de l'information terminologique. Chaque pôle constitue un secteur clé subdivisé selon les besoins en sous-secteurs.

\section{EXPLOITATION DE LA DOCUMENTATION}

Les fichiers-titres et les fichiers-auteurs de la Bibliothèque Dag Hammarskjöld en particulier, de même que les systèmes UNBIS et UNDIS, facilitent grandement l'accès à l'information documentaire.

Les informations réunies grâce à la recherche documentaire permettent ainsi à la Section de documentation et de terminologie, de répondre quotidiennement à un nombre croissant de problèmes ponctuels, de préparer des notes terminologiques susceptibles de satisfaire les besoins sans cesse nouveaux des traducteurs et des éditeurs et de publier des bulletins de terminologie multilingues, en vue d'obtenir rigueur et homogénéité dans les traductions, en particulier à l'occasion des grandes conférences, telles que le désarmement, le droit à la mer, la science et la technique au service du développement.

Si nous prenons l'exemple du dernier glossaire sur la Science et la Technique au service du Développement qui comprend 2827 termes en anglais, arabe, chinois, espagnol, français et russe, le choix du corpus a été délimité selon les critères suivants (domaines définis par les résolutions du Conseil économique et social 2028 (LXI) et 2035 (LXI) ) :

- alimentation et agriculture

- ressources minérales et énergétiques

- santé, établissements humains et environnement

- transports et communications

- industrialisation (y compris la production de biens d'investissement).

Les sources documentaires analysées ont été réparties en documents de l'Organisation des Nations unies, des institutions spécialisées et des sources extérieures.

\begin{tabular}{|c|c|c|}
\hline $\begin{array}{l}\text { ORGANISATIONS } \\
\text { Nations unies }\end{array}$ & $\begin{array}{l}\text { SOURCES PRIMAIRES } \\
-183 \text { rapports et } \\
\text { publications officielles } \\
-\quad 6 \text { glossaires } \\
-1 \text { thésaurus }\end{array}$ & $\begin{array}{l}\text { SOURCES SECONDAIRES } \\
-2 \text { bibliographies }\end{array}$ \\
\hline $\begin{array}{l}\text { Institutions } \\
\text { spécialisées }\end{array}$ & $\begin{aligned}-7 \text { rapports } \\
-10 \text { glossaires } \\
-\quad 1 \text { thésaurus }\end{aligned}$ & - 1 bibliographie \\
\hline $\begin{array}{l}\text { Sources } \\
\text { extérieures }\end{array}$ & $\begin{array}{c}-29 \text { dictionnaires } \\
\text { et glossaires } \\
-\quad 2 \text { encyclopédies } \\
-\quad 1 \text { thésaurus }\end{array}$ & - 1 bibliographie \\
\hline
\end{tabular}

Ainsi, tant sur le plan général de la documentation « pré-traduction » que sur le plan linguistique, pouvons-nous dégager l'importance vitale de la documentation. 